\title{
Más allá de la seguridad y la resiliencia. Hacia la intervención civil y el empoderamiento autógeno de las sociedades para construir su paz y su prosperidad
}

\author{
MANUel Ahedo*
}

\section{RESUMEN}

En la literatura sobre política y desarrollo internacional los conceptos de seguridad y resiliencia han tenido una creciente discusión y aplicación. La pregunta central es: ¿Qué puede hacer la comunidad internacional y específicamente los países centrales para avanzar hacia una seguridad humana integral, una resiliencia positiva y una prosperidad colectiva en las sociedades periféricas? Tras discutir críticamente la aportación de los dos conceptos, se relaciona la seguridad con la política internacional de intervención y de pacificación, y la resiliencia con las políticas de desarrollo; las dos relaciones son ilustradas con algunos casos empíricos. Sobre estas bases teóricas y analíticas se proponen los conceptos de intervención civil y de empoderamiento autógeno, como vías para avanzar hacia la seguridad humana integral y la resiliencia positiva. La intervención civil es la actuación de organizaciones civiles y de actores gubernamentales que colaboran de forma sincronizada y coordinada con instituciones públicas y civiles de la sociedad receptora para promover una seguridad y paz societal. El empoderamiento autógeno es la construcción simbiótica e interactiva de la resiliencia positiva impulsada exteriormente, percibido como propio. Estos dos conceptos son elementos de una teoría del desarrollo colectivo de una sociedad periférica en su contexto internacional.

\section{Palabras Clave}

Seguridad societal; resiliencia positiva; construcción de paz; relaciones globales de poder.

\section{TitLE}

Beyond security and resilience. Towards civil intervention and societies' autogenous empowerment to construct their peace and prosperity

\begin{abstract}
Within the literature on international politics and development the concepts of security and resilience have had growing analytical attention and application. The central question is: What can the international community and especially central countries do to foster a more integral human security, positive resilience and collective prosperity in peripherical societies? After critically discussing the contribution of the concepts of security and resilience, security is related to international humanitarian and peacemaking intervention and resilience to development; both relations are illustrated with some empirical cases. Based on these theoretical and empirical grounds, the concepts of civil intervention and autogenous empowerment are proposed, as ways to advance towards integral human security and positive resilience. Civil intervention refers to action by international civil organizations coordinated by public organizations collaborating with receiving societies' civil organizations to promote population's societal security and collective peace. Autogenous empowerment means the symbiotic and interactive construction of positive resilience promoted externally but perceived as own and endogenous. These concepts are part of a theory of collective development for a peripherical society within its international context.
\end{abstract}

\section{KEYWORDS}

Societal security; positive resilience; peace building; global power relations.

DOI:

https://doi.org//0.15366/relacionesinternacionales2020.43.003

Formato de citación recomendado:

AHEDO, Manuel,“Más allá de la seguridad y la resiliencia. Hacia la intervención civil y el empoderamiento autógeno de las sociedades para construir su paz y su prosperidad", en Relaciones Internacionales, n 43, 2020, pp. 49-67.

*Manuel

AHEDO, Profesor a tiempo parcial en los Departamentos de Ciencia Política y de Sociología de la Universidad de Copenhague (Dinamarca). Ha publicado en las áreas de sociología global y transnacional, y sociología de las relaciones internacionales. E-mail: manu. ahedo@gmail.com.

Recibido: 30/09/2019

Aceptado: I8/0I/2020

El autor quiere mostrar mi agradecimiento a los evaluadores por sus constructivas críticas y sugerencias. 


\section{$\mathrm{I}$}

\section{ntroducción}

Vivimos una época marcada por retos globales como el cambio climático y sus efectos en las poblaciones humanas, estructurales movimientos migratorios Sur-Norte y Sur-Sur, por terrorismo transnacional, inseguridades alimentarias, creciente desigualdad socioeconómica dentro de los países, mayor intensidad y confusión informativo-digital, conflictos violentos locales y regionales con complejas casuísticas entre factores étnicos, religiosos, recursos naturales, etc. En este contexto analizar cómo las comunidades humanas desarrollan vidas colectivas seguras y hacen frente a las adversidades requiere un elaborado aparato analítico. En las últimas décadas la idea de seguridad ha avanzado junto a la de resiliencia para entender cómo las sociedades organizadas en estados-nación construyen su seguridad integral interna y externa, y cómo sociedades afectadas por conflictos violentos, crisis, terrorismo, guerras, desastres naturales, etc. pueden superar los efectos negativos de los mismos. Los conceptos de seguridad y resiliencia integran múltiples aspectos y a la vez simplifican una realidad compleja.

Para tratar estas cuestiones a nivel global hay que superar tres sesgos teórico-analíticos: estadocéntrico, individualista y etnocéntrico'. El sesgo estadocéntrico deriva de la alta prioridad analítica dada al estado y de la creencia hegemónicamente aceptada en un estado real o potencialmente legítimo y tecno-racional ${ }^{2}$. El sesgo individualista viene del ideal ilustrado del ser humano con derecho a su libertad y moral individual y racional, sin prestar suficiente atención a las precondiciones societales colectivas para esas aspiraciones. El sesgo etnocéntrico promueve una bifurcación y asimetría analítica Norte-Sur y Oeste-Este³; es necesaria una redefinición de la deuda histórico-estructural de la región central con las regiones periféricas.

En la sección dos, desde una teoría social constructivista, empírica, reflexiva y crítica ${ }^{4}$ se discute la evolución de los conceptos de seguridad y resiliencia, en paralelo a la evolución de la realidad internacional y de las líneas generales de la política internacional; se presta atención a la acción exterior de la Unión Europea (UE), dada la responsabilidad histórica de Europa en el devenir del mundo y su papel en el actual orden mundial, crecientemente multipolar.

En la sección tres se extiende la discusión sobre seguridad y resiliencia a actuaciones e intervenciones en materia de política internacional, la cual es una cuestión central en la disciplina de Relaciones Internacionales. La seguridad se relaciona con la intervención internacional de pacificación y ayuda humanitaria en situaciones de inseguridad y conflicto violento, bajo el liderazgo de las Naciones Unidas (ONU) y de regiones centrales como la UE, y se ilustra con los conflictos del Congo y de Colombia. La resiliencia se relaciona con las políticas de desarrollo, y se ilustra con las políticas de ayuda de la UE a la zona del Sahel para hacer frente a la persistente sequía y sus efectos, y el movimiento global de la agricultura ecológica.

En la sección cuatro se proponen dos conceptos analíticos y normativos. Primero, respecto

\footnotetext{
Los sesgos estadocéntrico y etnocéntrico están presentes en la tradición teórica de las RRLL: DEL ARENAL, Celestino y SANAHUJA, José Antonio (coords.), Teorías de las Relaciones Internacionales, Tecnos, Madrid, 2015.

2 AHEDO, Manuel, “Una sociología histórica y global de la construcción del estado racionalizado de la modernidad internacional” en Relaciones Internacionales, $\mathrm{n}^{\circ}$ 38, 2018, pp. II-29.

3 En el texto se diferencia entre dos grandes regiones mundiales dentro de la separación Centro-Periferia: la región central o noroccidental y las diferentes regiones periféricas principalmente del Sur $y$ Este.

4 Por ejemplo, BARNES, Barry, The elements of social theory, UCL Press, Londres, 1995.
} 
a la intervención y ayuda humanitaria se propone el concepto de seguridad civil e intervención civil, enfatizando la acción de los actores civiles externos, principalmente las ONG humanitarias $(\mathrm{ONGH})$. Segundo, sobre el desarrollo de las sociedades periféricas se propone la idea de empoderamiento autógeno, que subraya las precondiciones para construir una resiliencia positiva desde una serie de acciones de origen externo, pero orientadas a estimular procesos internos, donde las ONG para el desarrollo (ONGD) juegan un importante papel.

En las conclusiones se resumen los argumentos principales y se incide en la importancia de concentrar el análisis en el contexto de los países no-centrales y periféricos, dentro de una perspectiva global. Esta perspectiva es necesaria asumiendo que estamos en una nueva era histórica global, caracterizada por el cambio del orden natural, como efecto del poder humano o Antropoceno y del poder de la economía capitalista o Capitaloceno.

\section{Seguridad y resiliencia: conceptualizaciones y aplicaciones}

Los polisémicos conceptos de seguridad y resiliencia han sido objeto de un intenso desarrollo y aplicación en varias direcciones. En esta sección se revisa la evolución y aplicación de los conceptos, y se enfatiza la dimensión colectiva o societal de los mismos.

\section{I.I. Seguridad: estado-sociedad-ciudadanía}

La seguridad, del latín securitas — cualidad de estar sin cuidado o no preocupado—, en su dimensión colectiva se puede manifestar de forma positiva, como la presencia de protección o prevención frente amenazas, y negativa, como la falta o ausencia de amenazas o riesgos potenciales. Los dos tipos de seguridad conforman un régimen de seguridad, que es la base intersubjetiva de la cultura de seguridad y de riesgo. En la época contemporánea la idea de seguridad ha evolucionado desde los enfoques de seguridad nacional o militar hasta las visiones más comprensivas e integrales sobre la seguridad societal o humana. En las últimas décadas, la seguridad humana ha sido considerada necesaria para promover un mundo más seguro y justo ${ }^{5}$.

Hasta el final de la Guerra Fría, la seguridad se centraba en la dimensión militar, la seguridad nacional o estatal recibía importantes recursos y era objeto central de legislación por los gobiernos. Era función del estado Hobbesiano, Westfaliano y Jánico (en referencia al dios griego Janus) mantener el orden interno respecto a las amenazas externas. Tras la caída del muro de Berlín y la progresiva desintegración del bloque soviético y la URSS una serie de cuestiones entraron en la agenda de seguridad: el terrorismo de inspiración fundamentalista, las tensiones nacionalistas y étnicas, los movimientos migratorios, los accidentes tecnológicos como el de la central nuclear de Chernóbil en 1986 en la actual Ucrania. El mundo, y de forma específica la UE, veía el surgimiento y expansión de estos viejos y nuevos tipos de problemas, derivados de conflictos étnico-identitarios, y diversos terrorismos con raíces identitarias o religiosas.

Un grupo de académicos europeos analizó estas realidades, destacando dos aspectos ${ }^{6}$. Primero, los conflictos entre países tenían un carácter diferente a la tensión capitalismo-comunismo, impulsados por un latente o explícito nacionalismo de diversa naturaleza política, religiosa, étnica,

\footnotetext{
NÚÑEZ, Jesús. et al., "Seguridad Humana, recuperando un concepto necesario" en Cuadernos del IECAH 7, Madrid, 2007.

BUZAN, Barry, People, states \& fear.An agenda for international security studies in the post-cold war era,The Open University, Londres, I982/I99।; WÆEVER, Ole, et al., Identity, migration and the new security agenda in Europe, Pinter Publishers, 1993.
} 
etc. Segundo, la globalización económica y comercial aumentaba el flujo transnacional de personas y objetos, y surgían problemáticas como el terrorismo internacional, migraciones descontroladas, etc. Desarrollaron la idea de la seguridad societal, a veces definida como intraestatal, centrada en los riesgos de seguridad del discurrir cotidiano de las sociedades. La seguridad societal aspiraba a identificar la base colectiva de seguridad entre el ciudadano/a y el estado, ya que la percepción de (in)seguridad y las prácticas de (auto)protección respecto a las amenazas suceden más allá de las opciones y posibilidades individuales y del estado.

En 1994, en el Informe sobre Desarrollo Humano del Programa de Naciones Unidas para el Desarrollo se proponía la seguridad humana como una forma de promover las libertades humanas desde la ausencia de necesidades, miedos o inseguridades, es decir, una seguridad centrada en el ser humano individual, objeto de la Carta Universal de los Derechos Humanos. El informe ampliaba horizontalmente la idea de seguridad e incluía siete tipos de seguridad: económica, alimentaria, de salud, ambiental, personal, comunitaria y política. Este informe supuso un cambio en la percepción de la acción humanitaria y la ayuda al desarrollo. El discurso respondía a la esperanza humanista y universal de la posguerra fría, pero adolecía de concreción y consenso definitorio para ser aplicado ${ }^{7}$.

Posteriormente se desarrolló el enfoque constructivista sobre la percepción de la amenaza, el riesgo y la necesidad de seguridad, en otras palabras, el proceso de securitización ${ }^{8}$. Los riesgos y la seguridad se conciben como una realidad construida, es decir, sobre una mínima base material y objetiva se construye una interpretación y percepción intersubjetiva y social respecto a la naturaleza de riesgo o amenaza de seguridad. Esta contribución enfatizaba la parte interpretativa de los riesgos, incluyendo factores sociales, culturales y políticos?

El atentado del II de septiembre del 200I en Nueva York alteró esta visión, y generó en la región central una estrategia militar contra el terrorismo, liderada por los Estados Unidos de América (EUA). Se reforzaron las visiones tradicionales de seguridad nacional y se priorizó la seguridad frente al desarrollo. En el 2003 la ONU reformuló su posición con el informe La seguridad humana ahora, que fortalecía la operatividad de la Comisión Permanente para la Seguridad Humana orientada a la intervención humanitaria y a la necesidad de quebrar el principio de soberanía territorial en casos de abuso a la población. En el 2004 la UE actualizó la Estrategia de Seguridad Europea del 2003 que apostaba por la seguridad humana, y publicó el informe Una doctrina de Seguridad Humana para Europa, que creaba una fuerza de intervención para establecer y para proteger o imponer la seguridad humana. La intervención o responsabilidad de proteger se convirtió en un objetivo prioritario.

Tras una década de inciertos resultados y preocupante evolución global, en 2016 la UE en su Estrategia Global de Política Exterior y de Seguridad ${ }^{10}$ dio un salto cualitativo hacia una agenda de

\footnotetext{
PÉREZ DE ARMIÑO, Karlos y MENDIA AZKUE, Irantzu (eds.), Seguridad Humana. Aportes críticos al debate teórico y político, Madrid, Tecnos, 2013.

8 BUZAN, Barry, et al. Security: a new framework for analysis, Boulder, Lynne Rienner, 1998.

9 BECK, Ulrich, World at risk, Polity, Londres, 2009.

10 UNIÓN EUROPEA: https://eeas.europa.eu/topics/eu-global-strategy_en [Consultado el 25 de septiembre de 2019].
} 
segunda generación sobre seguridad humana". Enmarca los conflictos violentos en condiciones sociales situadas históricamente, y reconoce la importancia la naturaleza contextual de los conflictos, la existencia de una sociedad civil enraizada como un actor clave, y la necesidad de integrar una diversidad de conocimientos y epistemes. Desde una mayor base empírica - reconoce la naturaleza híbrida e inestable de muchas situaciones de paz, y la importancia de las realidades locales contingentes-, la UE quiere ejercer un poder normativo global y promover que las leyes y los estados democráticos cumplan los derechos humanos y protejan a su ciudadanía ${ }^{12}$.

Los riesgos y las amenazas son diferentes según la región mundial. En la región central noroccidental los estudios de seguridad han analizado el terrorismo y los nuevos tipos de riesgos tecnológicos, epidémicos, salud ${ }^{13}$, etc., y el estudio de la seguridad ha adquirido un alto nivel de especialización, desarrollo teorizador y progreso empírico ${ }^{14}$. Se han desarrollado también los estudios $\operatorname{críticos}^{15}$, y se han propuesto posibles fertilizaciones cruzadas entre los diferentes enfoques. Newman ${ }^{16}$ sugirió integrar los estudios críticos de seguridad y la seguridad humana en la línea propuesta por la ONU. Burgess ${ }^{17}$ propuso una amplia sociología de la seguridad en la actual sociedad tecnológica y de (des)información, estudiando el malestar, el desasosiego y del miedo, analizando las redes, flujos y contenidos de influencia, de información, respecto a la presencia y la ausencia de acción política o de gobierno sobre seguridad.

En muchas regiones periféricas la seguridad de la población es un problema estructural, relacionado con resolución de conflictos violentos, pacificación y estabilidad institucional y política, como por ejemplo el desarrollo de unos efectivos y legítimos cuerpos de seguridad ${ }^{18}$. La inseguridad alimentaria también ha aumentado en muchos países. La idea de seguridad civil incluye todos estos riesgos básicos colectivos, y constituye una precondición para avanzar hacia la idea de seguridad humana integral.

En suma, en las regiones periféricas la necesidad es promover la seguridad civil colectiva de la población — física, alimentaria, ausencia de violencia, etc.- la cual debe concebirse como un derecho colectivo a un mínimo de estabilidad ontológica sin altos niveles de incertidumbre respecto a riesgos o amenazas. La seguridad civil es un proceso de corresponsabilidad a varios niveles: a) de la propia sociedad o comunidad desde su cultura de riesgo y seguridad, b) del estado como institución política detentora de los poderes y recursos para mantener la seguridad de la población en su territorio;y c) del orden internacional y de sus estructuras de poder y dominación.

\footnotetext{
" MARTIN, Mary y OWEN Taylor, "The Second Generation of Human Security: Lessons from the UN and EU Experience" en International Affairs, $\mathrm{n}^{\circ}$ 86. I, 2010, pp. 2II-224.

12 KALDOR, Mary, RANGELOV, lavor y SELCHOW, Sabine (eds.), EU Global Strategy and Human Security, Routledge, Londres, 2018.

13 BURGUESS, J. Peter, The Future of Security Research for the Social Science and Humanities, European Science Foundation, Estrasburgo, 2014.

14 VERDES-MONTENEGRO ESCANÉZ, Francisco J., "Securitización: agendas de investigación abiertas para el estudio de la seguridad" en Relaciones internacionales, $\mathrm{n}^{\circ}$ 29, 2015, pp. III-132.

15 VAUGHAM-WILLIAMS, Nick, Critical security studies:An introduction, Routledge, Oxford, 2010.

16 NEWMAN, Edward, “Critical Human Security Studies” en Review of International Studies, n 36, 2010, pp. 77-94.

17 BURGUESS, J. Peter, “Security Studies” en GUILLAUME, Xavier y BILGIN, Pinar (eds.), Routledge handbook of international political sociology, Routledge, Londres, 2016.

${ }^{18}$ En países claramente inseguros, especialmente en África y Asia —Irak, Afganistán, etc.— MURRAY, Tonita, "Police-building in Afghanistan: A case study of civil security reform" en International Peacekeeping, n I4.I, 2007, pp. 108-126.
} 


\section{I.2. Resiliencia: ¿Tradicional o positiva? ¿Estatal o societal?}

La palabra resiliencia, del latín resilio — saltar para atrás-, ha sido entendida con dos significados principales: a) la tradicional, como capacidad de persistencia o resistencia, y b) la positiva, como capacidad de sobreponerse a los problemas y adaptarse a las nuevas situaciones. En los dos significados la idea de resiliencia es aplicable a múltiples situaciones, y en diferentes áreas de conocimiento ha servido como metáfora inspiradora para comprender esa capacidad individual y colectiva de resistencia, superación y adaptación. Sin embargo, la idea de resiliencia no supone una contribución realmente original al conocimiento de la dinámica de la vida humana colectiva. La sociología y la antropología han enfatizado dos aspectos: a) la fuerza de las tradiciones en la vida humana colectiva, aunque estas puedan incluso ser negativas para el propio colectivo; $y$ b) la capacidad de adaptación de los colectivos humanos a los cambios del entorno, y de sobrevivir y sobreponerse a las adversidades. En el campo de las relaciones internacionales el concepto de resiliencia ha sido objeto de un intenso desarrollo teorizador y aplicado ${ }^{19}$. La idea de resiliencia se ha usado para analizar diversas realidades ${ }^{20}$, y se pueden identificar tres perspectivas: analíticaescéptica, crítica-idealista y analítica-aplicada.

En una perspectiva analítica escéptica, los sistemas sociales, y también los naturales, se perciben como resilientes, difíciles de cambiar, evolucionando a su propio ritmo y lógica. Respecto a la resiliencia tradicional y su estructura social de poder latente, una cuestión clave es ¿cuál es la institución y el grupo social más interesado en preservar esa estabilidad? Los grupos sociales que controlan los estados están interesados en defender su poder y status-quo. La resiliencia tradicional general de una sociedad esconde una resiliencia parcial indirecta e interesada en torno al estado, la cual es una construcción social que se puede deconstruir.

En una perspectiva crítica inspirada en teorías idealistas-discursivas, por ejemplo, de Foucault, la resiliencia se ha considerado parte del discurso neoliberal que reduce y relativiza la responsabilidad internacional de los estados y países centrales ${ }^{21}$. Es decir, la resiliencia forma parte del modo o régimen neoliberal de mentalidad de gobernar — governmentality — basado en el autogobierno impuesto indirectamente por la autoridad estatal liberal ${ }^{22}$. Enfatizar la importancia de la resiliencia de las sociedades periféricas es una forma de desinternacionalizar y de justificar el no-intervencionismo de los países centrales ${ }^{23}$. De manera sutil, la resiliencia ha estimulado la concepción de los conflictos, del subdesarrollo y del lento y frustrante proceso de democratización como fenómenos endógenos, indicando así la correspondiente responsabilidad de las propias sociedades y estados respecto a sus amenazas e inseguridades. En suma, el discurso de la resiliencia esconde un efecto de despolitización y desinternacionalización por parte del nuevo paradigma noroccidental neoliberal de no intervención o limitado intervencionismo en las relaciones internacionales.

\footnotetext{
19 CHANDLER, David y COAFFEE, Jon, The Routledge handbook of international resilience, Routledge, 2016.

${ }^{20}$ LANGA MARTíNEZ, Laura, "El camino a la resiliencia. La llave para la convergencia de enfoques y actores", Documento I5/20/3, IECAH, Madrid.

${ }^{21}$ REID, Julian, "Interrogating the neoliberal biopolitics of the sustainable Development-Resilience Nexus" en International Political Sociology, $\mathrm{n}^{\circ}$ 7.4, 2013, pp. 353-367.

22 JOSEPH, Jonathan, "Resilience as embedded neoliberalism: a governmentality approach" en Resilience: International Policies, Practices and Discourses, $\mathrm{n}^{\circ}$ I:I, 2013, pp. 38- 52.

${ }^{23}$ CHANDLER, David, “International state-building and the ideology of resilience” en Politics, $n^{\circ}$ 33.4, 2013, pp. 276-286.
} 
En una perspectiva analítica aplicada y de intervención se arguye que los colectivos humanos afectados por problemas necesitan estímulo y ayuda para desarrollar su capacidad de resistencia, superación y adaptación. Al hablar de resiliencia se enfatizan las capacidades para la predisposición y la preparación ante los posibles riesgos y amenazas, y la disposición a explotar las posibles oportunidades que surgen en los desastres. En lugar de prevenir los riesgos, una estrategia resiliente puede ser prepararse de forma constructiva para convivir con los riesgos, muchos de ellos imposibles de conocer y predecir. La cultura de resiliencia resulta de una colaboración entre estado y sociedad. En la ayuda al desarrollo el estado suele ejercer una importante tarea de filtración e intermediación. Sin embargo, la resiliencia positiva colectiva y societal surge de la interacción entre estado y sociedad, generalmente a iniciativa de la sociedad civil, en sus diversas formas de organización ${ }^{24}$.

En suma, se argumenta que la construcción de la resiliencia positiva y proactiva debe fundamentarse en la sociedad civil, la cual con el tiempo debe empujar al estado a aceptar su responsabilidad en la capacitación de su ciudadanía. ¿Cómo aprende un colectivo humano la capacidad de sobreponerse a los problemas, desastres y adversidades? Primero, se aprende a superar problemas de forma involuntaria desde experiencias previas, y voluntaria tomando riesgos y asumiendo sus efectos y anticipando problemas. Segundo, es el resultado de compartir experiencias y prácticas; las instituciones públicas deben facilitar los mecanismos para la distribución de conocimiento y experiencia. Tercero, la resiliencia positiva se construye a nivel local en un marco multinivel dentro del estado-nación y de la región o civilización —área subsahariana, islámica, América Latina, etc.-.

\section{Seguridad e intervención internacional. Resiliencia y desarrollo}

Martinussen ${ }^{25}$ recogió el debate sobre la relación entre seguridad y desarrollo de las sociedades del tercer mundo o periféricas. La seguridad y la resiliencia responden de forma complementaria a las dinámicas de intervención y desarrollo internacional, como una actualización del continuum emergencia-desarrollo ${ }^{26}$ o seguridad-desarrollo ${ }^{27}$. Algunos estudios de casos occidentales han integrado los dos conceptos desde un enfoque performativista ${ }^{28}$. Sin embargo, para analizar la realidad de los países periféricos se considera necesario distinguir dos relaciones analíticas: a) la relación entre seguridad y política internacional, respecto a conflictos armados y violentos, y a las prácticas de intervención exterior a través de las $\mathrm{ONGH}$ y b) la resiliencia está altamente relacionada con las políticas de ayuda al desarrollo, con la participación de las ONGD.

Tradicionalmente la intervención internacional en los países periféricos se ha orientado

${ }^{24}$ KRÜGER, Marco, "Building Instead of Imposing Resilience: Revisiting the Relationship Between Resilience and the State" en International Political Sociology, n 13.1, 2018, pp. 53-67.

${ }^{25}$ MARTINUSSEN, John Degnbol, Society, state \& market. A guide to competing theories of development, MSO, Danish Association for International Cooperation, Copenhague, 1997.

${ }^{26}$ PÉREZ DE ARMIÑO, Karlos, La vinculación emergencia-desarrollo en el marco del nuevo humanitarismo. Reflexiones y propuestas, Coordinadora de ONG para el Desarrollo, Madrid, 2002.

27 SANAHUJA, José Antonio y SCHÜNEMANN, Julia, "El nexo seguridad-desarrollo: entre la construcción de la paz y la securitización de la ayuda" en PÉREZ DE ARMIÑO, Karlos y MENDIA AZKUE, Irantzu (eds.), Seguridad Humana. Aportes críticos al debate teórico y político, Madrid, Editorial Tecnos, 2013;YOUNGS, R. La fusión entre seguridad y desarrollo: ¿Otro estancamiento europeo? FRIDE, Madrid, 2007.

${ }^{28}$ BRASSET, James y VAUGHAN-WILLIAMS, Nick, "Security and the performative politics of resilience: Critical infrastructure protection and humanitarian emergency preparedness" en Security Dialogue, n 46. I, 2015, pp. 32-50. 
hacia el estado y los gobiernos. Sin embargo, ante los pobres resultados, se sugiere que la acción exterior gubernamental y de las ONG puede priorizar la sociedad civil y las comunidades locales para que al mismo tiempo que se empoderan se conviertan en motor del cambio de los estados y los gobiernos hacia mayores estándares en derechos humanos, democracia y seguridad. La participación de las ONG en la acción-intervención en las sociedades civiles y locales puede generar una relación bilateral más simétrica y paritaria. Este argumento se basa en una visión sociológica del estado: desde un marco mínimo de libertades civiles las fuerzas sociales en coordinación presionan directa e indirectamente para la democratización y la mejora del estado y del sistema político.

\section{I. Seguridad e intervención internacional: ayuda exterior a la construcción local de la seguridad civil}

¿Cuál puede ser por parte de instituciones públicas internacionales la forma de intervención más efectiva para ayudar a solucionar conflictos y aumentar la seguridad? La respuesta es que el objetivo debería centrarse en la seguridad civil o poblacional,y en la promoción de los procesos de construcción de la paz y la seguridad a nivel local y comunitario. La seguridad básica, como ausencia de amenaza violenta, ha sido considerada un objetivo prioritario de la gestión de los conflictos bélicos o violentos. La seguridad civil en muchos conflictos puede depender de intereses tanto latentes y manifiestos, como no declarados y latentes, que pueden ser locales - grupos de poder estatal, élites de las comunidades locales, relaciones intergrupales, etc.—y globales — geopolítica, industria armamentística, empresas globales de recursos naturales, etc. - - Los conflictos violentos a gran escala se caracterizan por una compleja concatenación de estructuradas dinámicas locales. Ese puzle contingente de intereses y estrategias dificulta una efectiva intervención exterior pacificadora. Para superar esa limitación se sugiere una mayor focalización en la construcción local o civil de la paz y la seguridad por los actores públicos y civiles exteriores, superando la tradición estadocéntrica de las relaciones estatales y gubernamentales y las limitaciones para entender la complejidad local de los conflictos.

Las ONG especializadas en acción humanitaria ${ }^{29}$ tienen unas características de neutralidad e imparcialidad que pueden ser claves en procesos de pacificación y de construcción de la seguridad civil de la población. Se ha propuesto que estas $\mathrm{ONGH}$ colaboren más en las misiones de paz, junto a fuerzas militares de pacificación ${ }^{30}$. Especial relevancia tienen las ONGH de los países medios o pequeños, como es el caso de muchos de la UE, para promover procesos de construcción de paz estable la seguridad civil ${ }^{3 !}$.

Estos argumentos teóricos ayudan a entender dos casos empíricos de conflictos históricos y de gran escala: a) el conflicto del Congo, dentro de la Región de los Grandes Lagos del este subsahariano, y b) el conflicto de Colombia y los recientes acuerdos de paz de 2016.

La región de la República del Congo y sus países limítrofes acumula una historia de más de

\footnotetext{
${ }^{29}$ Acción Humanitaria; concepto y evolución, en Diccionario de Acción Humanitaria y Cooperación al Desarrollo de HEGOA: http://www.dicc.hegoa. ehu.es/listar/mostrar/I [Consultado el 25 de septiembre de 2019].

${ }^{30}$ ABIEW, Francis K.,"NGO-military relations in peace operation" en International Peacekeeping, n I0, I, 2003, pp. 24-39; SAUQUILLO, Francisca, "La otra cara de la resolución de conflictos. Las ONG" en Cuadernos de estrategia, n 147, 2010, pp. I4I-173.

${ }^{31}$ RUTHERFORD, Ken, et al. Reframing the agenda: the impact of NGO and middle power cooperation in international security policy, Greenwood Publishing Group, 2003.
} 
cincuenta años de conflictos y violencia desde los procesos de independencia de los años sesenta ${ }^{32}$. En la República del Congo el conflicto ha sufrido dos guerras - 1996-1997 y 1998-2002/03-. La segunda guerra, también llamada la Gran Guerra Africana o Guerra Mundial Africana, provocó más de 4 millones de muertos directos o indirectos, y unos dos millones de desplazados. En este conflicto se han enfatizado dos cuestiones: a) la de seguridad, por los muchos grupos armados y violentos; $y$ b) los intereses nacionales e internacionales respecto a algunos minerales ${ }^{33}$. Ambas cuestiones han definido el conflicto a un nivel estatal e internacional, marginando la complejidad local y el papel y potencialidad de la sociedad civil.

En la cuestión de la seguridad ha habido una fuerte privatización, en colaboración con el estado y las fuerzas policiales ${ }^{34}$. La seguridad en muchos países periféricos ha recibido soluciones neoliberales, y el auge de las empresas de seguridad en tareas de seguridad pública ha sido una constante en las últimas décadas ${ }^{35}$. En Congo la privatización ha alcanzado altos niveles, y el estado ha perdido su razón de ser como institución con legitima posesión de la violencia en base a los principios de seguridad y de representación de la mayoría.

En la cuestión de los recursos naturales, la extracción y circulación de minerales como coltán, casiterita, wolframio y oro, usados en la industria microelectrónica, está controlada por grupos armados privados y públicos. La legislación transnacional para regular y controlar su importación y aplicación es lenta y compleja. La ONU ha iniciado la creación de una convención al respecto. El gobierno de los EUA ha regulado la información sobre la provisión de materiales de las empresas, pero sin un esquema claro de control y sanción. La UE aprobó en 2017 una regulación sobre la cadena de provisión de importadores de algunos metales desde áreas en conflicto, a entrar en vigor en enero del $2021^{36}$,y en 2018 publicó una directiva no-vinculante para la identificación de las áreas de conflicto a ser añadida a la regulación de 2017.

Por el contrario, algunos autores como Autesserre ${ }^{37}$ han subrayado la complejidad local del conflicto, y la necesidad de mejorar el diagnóstico y las prácticas de intervención pacificadora: The international actors' concentration on trafficking of mineral resources as a source of violence has led them to overlook the myriad other causes, such as land conflict, poverty, corruption, local political and social antagonisms, and hostile relationships between state officials, including security forces, and the general population (p. 205).

32 GAHAMA, Joseph “Une cinquantaine d'années de conflits et de violences dans la région des Grands Lacs africains" en LUMUMBAKASONGO Tukumbi \& GAHAMA Joseph (eds.), Peace, Security and Post-conflict Reconstruction in the Great Lakes Region of Africa, CODESRIA, Dakar (Senegal), 2017.

33 LE BILLON, Philippe, Wars of Plunder: Conflicts, Profits and the Politics of Resources, Oxford University Press, 2014.

34 TUKALA, Célestin T., "Etats des Grands Lacs et privatisation de la sécurité: quel enjeu pour la gouvernance?” en LUMUMBA-KASONGO Tukumbi \& GAHAMA Joseph (eds.) Peace, Security and Post-conflict Reconstruction in the Great Lakes Region of Africa, CODESRIA, Dakar (Senegal), 2017.

35 ABRAHAMSEN, Rita y WILLIMAS, Michael C., Security beyond the state: Private security in international politics, Cambridge University Press, 20 I0; MEAGUER, Kate, "The strength of weak states? Non-state security forces and hybrid governance in Africa" en Development and Change, $\mathrm{n}^{\circ}$ 43.5, 20I2, pp. I073-II0I.

${ }^{36}$ UNIÓN EUROPEA, Responsible trade in minerals from high-risk or conflict areas: https://eur-lex.europa.eu/legal- content/EN/ LSU/?uri=CELEX:320 I7R082 I \&qid= I542740800246 [Consultado el 27 de septiembre de 2019].

37 AUTESSERRE, Séverine, “Dangerous tales: Dominant narratives on the Congo and their unintended consequences" en African Affairs, $\mathrm{n}^{\circ}$ III.443, 20I2, pp. 202-222. 
Para Arnould y Valasserot ${ }^{38}$, los principales errores de la UE en su intervención en Congo han sido dos: a) una excesiva prioridad en la construcción de instituciones políticas formales sin contar con las necesarias precondiciones sociales; $y$ b) una marginación de su tarea diplomática y de mediador. En suma, en el conflicto en el Congo es necesaria una mayor amplitud de visiones y perspectivas, más enraizadas empírica y localmente. Desde este conocimiento, las $\mathrm{ONGH}$, con diferentes focos de atención, pueden enfatizar diferentes aspectos del conflicto para abarcar su complejidad, mientras se presentan como legítimos y aceptados mediadores.

El conflicto de Colombia tiene unos antecedentes desde mediados del siglo XX. La irrupción de guerrillas revolucionarias inspiradas en diferentes referencias socialistas -FARC y ELN han sido los grupos más relevantes- generó una larga guerra civil, complejizada por cuestiones territoriales y sociales, por la producción y comercialización de cocaína, y por el surgimiento y desarrollo de grupos paramilitares. Los intentos de alcanzar la paz comenzaron en la década de los setenta y ochenta.Tras diferentes estrategias, negociaciones y fracasos, en 2016 se firmaron los acuerdos de paz entre el gobierno y las FARC, y su posterior plebiscito de refrendo. Este acuerdo de paz ha sido el resultado de un proceso interno y externo. Dentro del país, el proceso se ha fundamentado en unas claras dinámicas nacionales y locales, en el que se destaca el papel de algunos líderes políticos y guerrilleros. Fuera del país, los actores internacionales han ejecutado de manera efectiva un comedido papel secundario ${ }^{39}$.

Además de estos aspectos se ha subrayado el importante proceso civil y comunitario en la construcción de la paz con ayuda exterior. La sociedad civil colombiana ha sido un actor estable y presente ${ }^{40}$, especialmente en los momentos críticos donde el proceso parecía afrontar obstáculos y crisis, como la Audiencia Nacional por la Paz y el Pacto Ético de las mujeres en 2013, y la reactivación del Consejo Nacional de Paz en 20I5. En la dimensión local y civil un aspecto clave fue la promoción de los Laboratorios de Paz, dentro de la Ayuda al Desarrollo de la UE ${ }^{41}$. La UE optó por ayudar a construir la paz desde abajo, en cooperación con la sociedad civil local, apoyando a las comunidades locales a transformar las bases democráticas del estado.

Estos dos casos contribuyen a un mejor entendimiento de la construcción de la seguridad y de la paz en situaciones de conflictos y clara inseguridad, desde una perspectiva contingente $y$ civil y viendo el proceso de paz como empoderamiento, tal y como han desarrollado en España y en castellano autores como Fisas, Muñoz y Bolaños, etc ${ }^{42}$. Desde los muchos ejemplos de fracaso

\footnotetext{
${ }^{38}$ ARNOULD, Valerie $y$ VLASSEROOT, Koen "EU policies in the DR Congo: Misaligned ambitions" en KALDOR, Mary, RANGELOV, lavor $y$ Sabine SELCHOW (eds.), EU Global Strategy and Human Security, Routledge, Londres, 2018.

39 BORDÁ, Sandra y GÓMEZ, Santiago "La Internacionalización del proceso de paz de la Habana: del aislacionismo a la contención" en TICKNER, A. y BITAR, S. (ed.), Nuevos enfoques para el estudio de las relaciones internacionales en Colombia, Universidad de los Andes, Bogotá, 2017; CUAJANTE, Ximena, “La comunidad internacional y su participación en los procesos de paz en Colombia” en Equidad y Desarrollo, $n^{\circ}$ 26, 20I6, pp. 207-222.

40 GONZALEZ, Catherine, "Iniciativas de paz en Colombia" en Civilizar: Ciencias Sociales y Humanas, n 10.18, 2010, pp. 35-53; HERNANDEZ DELGADO, Esperanza, “Negociaciones de paz en Colombia: una mirada en perspectiva de construcción de paz” en Papel Politico, 21.1, 2016, 35-56; MAHECHA, Jimena, «Iniciativas locales de paz: tres casos desde la resistencia civil para la reflexión» en Ciencia Política, $n^{\circ}$ 13.26, 2018, pp. 153-181.

${ }^{41}$ CASTAÑEDA, Dorly, ¿Qué significan los laboratorios de paz para la Unión Europea?" en Colombia internacional,I n 69, 2009, pp. 162-179; CASTAÑEDA, Dorly, “The European Union in Colombia: Learning how to be a peace actor", L'Institut de Recherche Stratégique de l'École Militaire, Paris, 2012

42 FISAS, Vicenç, Introducción a los procesos de Paz, Barcelona: Escola de Cultura de Pau, 2010; MUÑOZ, Francisco A. y Jorge BOLAÑOS
} 
de pacificación, Autesserre ${ }^{43}$ arguye que el éxito en obtener una paz estable radica en el nivel local de los conflictos, y reconoce que la ONU ha comenzado a aceptar que la construcción de paz estable requiere de una importante participación de la población local, ya que la comunidad local tiene conocimiento y experiencias sobre lo que mejor puede promover la paz. Auteserre también enfatiza las prácticas organizativas e institucionales de los actores exteriores, a la vez que sugiere el potencial de las $\mathrm{ONGH}^{44}$. Las $\mathrm{ONGH}$ tienen un estatus adecuado para apoyar a ONG pacifistas locales para dos objetivos: a) impulsar la construcción de la pacificación y la seguridad ${ }^{45}$; y b) facilitar la participación de las comunidades locales, más o menos representadas por sus organizaciones civiles, en los procesos de acuerdo y reconciliación.

En suma, la seguridad humana integral debe ser impulsada y promovida,y debe fundamentarse en una seguridad civil, la cual apela a la responsabilidad de la comunidad internacional y las agencias intergubernamentales. Hay tres aspectos claves. Primero, el carácter colectivo, societal y civil de la seguridad. Segundo, la responsabilidad estatal interior y exterior debe orientarse a la seguridad de la población; las fuerzas de seguridad —policía y ejércitos- deben legitimar su función y actividad en su aceptación por la mayoría de la ciudadanía. Tercero, se sugiere el desarrollo de una orientación civil en la intervención pública exterior y una mayor intervención a cargo de las $\mathrm{ONGH}$. Una situación de paz estable requiere unos fundamentos societales y locales, desde capacidades de empoderamiento locales ${ }^{46}$.

\subsection{Resiliencia, desarrollo y ayuda al desarrollo: construyendo la resiliencia positiva}

En la revisión de las teorías de desarrollo internacional que realizó Martinussen ${ }^{47}$ se observa que frente a las teorías dominantes centradas en los mercados y en los estados, desde los análisis críticos de autores como Gran y Robertson ${ }^{48}$, las teorías societales o civiles de desarrollo son un necesario contrapeso al papel de los estados y los mercados. Estas teorías argumentan que las políticas y programas de desarrollo están avocadas al fracaso, si la sociedad, en su vertiente civil o popular, no participa de forma activa y sustantiva en esas políticas y programas. La ayuda al desarrollo orientada a la sociedad civil y las comunidades locales ha ido ganando importancia.

En las últimas décadas el desarrollo humano se concibe con un fuerte fundamento social y societal. Sen ${ }^{49}$ lo concibió como el ejercicio de la libertad individual basada en una serie de capacidades para tomar decisiones y opciones, y para definir el modelo de vida digna. Esas capacidades solo pueden ser ofrecidas y desarrolladas de manera general por instituciones públicas y civiles. De hecho, muchos de los países periféricos necesitan decidir colectivamente qué tipo de

CARMONA (eds.), Los habitus de la paz. Teorías y prácticas de la paz imperfecta, Editorial Universidad de Granada, 201 I.

43 AUTESSERRE, Séverine, “International peacebuilding and local success:Assumptions and effectiveness” en International Studies Review, $\mathrm{n}^{\circ}$ I9.I, 20I7, PP. II4-I32.

44 AUTESERRE, Sverine, Peaceland: Conflict resolution and the everyday politics of international intervention, Cambridge University Press, 20 I4.

45 DONAIS, Timothy, “Empowerment or Imposition? Dilemmas of Local Ownership in Post-Conflict Peacebuilding Processes" en Peace \& Change, n 34.I, 2009, pp. 3-26; DONAIS, Timothy, Peacebuilding and local ownership: Post-conflict consensus-building, Routledge, Londres, 2012.

46 DE CONING, Cedric, "From peacebuilding to sustaining peace: Implications of complexity for resilience and sustainability" en Resilience, $\mathrm{n}^{\circ}$ 4.3, 2016, pp. I66-18I.

47 MARTINUSSEN, John D., Society, state... op. cit.

48 GRAN, Guy, Development by people; citizen construction of a just world, Praeger, 1983; ROBERTSON, Alexander F., People and the State: An Anthropology of Planned Development, Cambridge University Press, 1984.

49 SEN, Amartya, Development as freedom, Anchor Books, 1999. 
desarrollo colectivo desean. En la era del cambio climático todas las sociedades deben redefinir su futuro material, de consumo y estilo de vida. La naturaleza de muchos países periféricos es clave para reducir el nivel de $\mathrm{CO} 2$ en la atmosfera. Si el desarrollo se define como el patrón material y económico que ha seguido la región noroccidental, su contribución ecológica será inferior. La resiliencia positiva puede ayudar a que las sociedades periféricas resistan también las influencias normativas del exterior y sean capaces de hacer sus propias definiciones, tomar sus decisiones y prácticas colectivas acordes con su entorno, su marco de necesidades y sus visiones de futuro. Las ONGD deben priorizar el desarrollo de la capacidad de interpretación y de decisión de esas sociedades y comunidades.

¿Pueden realmente las ONGD empoderar a las sociedades en desarrollo? Martinussen recogía un debate incipiente sobre la forma en que los actores externos o extranjeros pueden ayudar a la sociedad o comunidad local a ser un actor activo y sustantivo en su propio desarrollo. En aquella época la literatura empírica era crecientemente escéptica sobre la efectividad de los actores externos a la hora de generar un empoderamiento societal y civil en la sociedad receptora ${ }^{50}$. Las pocas voces optimistas subrayaban principalmente el posible papel que las ONG u organizaciones civiles de los países donantes podían tener a la hora de promover liderazgos comunitarios y civiles locales, que a su vez pudieran estimular y promover el empoderamiento local del desarrollo desde la cercanía y la legitimidad cultural ${ }^{5 !}$.

Se traen aquí dos casos ilustrativos de cómo la resiliencia puede ser entendida y aplicada:a) una aplicación activa, en el desarrollo de la agricultura agroecológica promovida por el movimiento social global Via Campesina, que ha contado a nivel internacional con el apoyo indirecto de la política de la UE respecto al medio ambiente y los organismos modificados genéticamente OMG—; y b) una aplicación distante, en la iniciativa de la UE respecto al Sahel.

La llamada revolución agroecológica se puede entender como un movimiento social multinivel e interseccional, basado en el empoderamiento de las comunidades campesinas ${ }^{52}$. Dentro del movimiento social, comunidades científicas o investigadoras, gobiernos locales, comunidades indígenas, ONG, etc. colaboran en la promoción de una mayor soberanía alimentaria, desarrollo sostenible y solidaridad global interlocal ${ }^{53}$. Destaca la labor de Via Campesina, creada en 1993, presente en todas las regiones del mundo con una relativa mayor presencia en América Latina y África, cuenta con un relativo fuerte apoyo europeo -26 de las 182 organizaciones $^{54}$ _, y su objetivo principal ha sido la promoción de un modelo agrícola sostenible, local y orientado social y comunitariamente. ¿Qué intervención internacional central ha ayudado a este movimiento global? Se puede argüir que la UE ha contribuido indirectamente a la promoción de la agricultura

50 KORTEN, David C., Getting to the 2 I st century:Voluntary action and the global agenda, Kumarian Press, 1990.

5I GRAN, Guy, Development by ... op.cit.

52 ALTIERI, Miguel A. y TOLEDO,Victor Manuel, “The agroecological revolution in Latin America: rescuing nature, ensuring food sovereignty and empowering peasants" en The Journal of Peasant Studies, $n^{\circ} 38.3$, 20I I, pp. 587-6I 2.

53 WALSH-DILLEY, Marygold, WOLFORD, Wendy y MCCARTHY, James, "Rights for resilience: food sovereignty, power, and resilience in development practice" en Ecology and Society, 21.1, 2016: www.jstor.org/stable/26270348 [Consultado el 25 de septiembre de 2019 ].

54 “La Vía Campesina es un movimiento de masas de base cuya vitalidad y legitimidad proviene de las organizaciones campesinas a nivel de base. El movimiento se basa en la descentralización del poder entre todas sus regiones. La secretaría internacional gira de acuerdo con la decisión colectiva realizada cada cuatro años en la Conferencia Internacional; su sede ha estado en Bélgica — 1993-1996_, Honduras — 1997-2004-, Indonesia -2005-20I3-,y desde 2013 en Harare, Zimbabue": www.vicampesina.org [Consultado el 25 de septiembre de 20 I9]. 
ecológica a través de un cuestionamiento y prohibición de los $\mathrm{OMG}^{55}$, a pesar de la fuerte presión industrial y científica para superar la postura crítica y de precaución, y pasar a una regulación positiva de los $\mathrm{OMG}^{56}$. La UE ha asumido asimismo su responsabilidad internacional en materia medioambiental y ha liderado las políticas contra el cambio climático. Se puede afirmar que dentro de los discursos dominantes globales estas políticas de la UE han ayudado de forma indirecta a la legitimidad y el desarrollo de la agroecología en los países periféricos.

El caso de resiliencia distante se refiere a las políticas de la UE hacia la región del Sahel subsahariano, y específicamente la zona del Cuerno de África, con relación a las sequías y sus correspondientes hambrunas. En 20II este desastre fue objeto de atención internacional ${ }^{57}$. En la UE se planteó el problema inspirado en la idea de resiliencia. En 2012 propuso el UE SHARE: Supporting the Horn of Africa's Resilience ${ }^{58}$ y en el 2012-2013 presentó una iniciativa más ambiciosa: Alliance Globale pour l'initiative Résilience ${ }^{59}$ o AGIR - Sahel, vigente hasta hoy ${ }^{60}$. La UE aspiraba a construir capacidades locales con una perspectiva a largo plazo, a modo de gobernanza a distancia ${ }^{61}$, y de una forma pragmática con unos principios generales de actuación ${ }^{62}$. Pero la realidad del Sahel es compleja. Además del factor natural, el sistema agrícola, ganadero y de alimentación es clave para afrontar el problema. Por la parte académica e institucional, Shiferaw et al. ${ }^{63}$ indican que es necesario una mejora de las opciones tecnológicas, de políticas públicas e institucionales para que los agricultores y ganaderos puedan afrontar decisiones propias de riesgo controlado o compartido para adaptar el modelo agropecuario a las nuevas condiciones climáticas y naturales. Estos autores identifican dos niveles de desarrollo de estas soluciones. En un nivel directo y próximo están las opciones de nuevos tipos de maíz más resistentes a la sequía, métodos de conservación de agua, prácticas productivas, sistemas de previsión y comunicación del tiempo y de la sequía, sistemas de aseguración de las cosechas respecto al clima, desarrollos de mecanismos para establecer precios acordes con el esfuerzo y dedicación productiva, etc. En un nivel más sistémico, estas propuestas deberían enmarcarse en un sistema integral de protección de la naturaleza y de los ecosistemas, de políticas públicas de protección social y de alimentación. Desde una postura crítica y alternativa, actores como Via Campesina defienden que estas soluciones se complementen con iniciativas de agricultura ecológica, no monocultivo y soberanía alimentaria, desde la experimentación local ${ }^{64}$.

55 FALKNER, Robert "The political economy of 'normative power' Europe: EU environmental leadership in international biotechnology regulation" en Journal of European Public Policy, n 14.4, 2007, pp. 507-526.

${ }^{56}$ DAVISON, John y AMMANN, Klaus, "New GMO regulations for old: determining a new future for EU crop biotechnology” en GM crops \& food, $n^{\circ}$ 8.1, 2017, pp. 13-34.

57 GUBBELS, Peter, “Escaping the Hunger Cycle: Pathways to resilience in the Sahel” en Oxfam Policy and Practice:Agriculture, Food and Land, $\mathrm{n}^{\circ}$ II.6, 20II, pp. 165-288.

${ }^{58}$ CONTRERAS, L.,"La resiliencia de la teoría a la práctica: un enfoque para romper el círculo del hambre” Cap. 7 en el Informe del Observatorio de la Acción Humanitaria, IECAH, Madrid, 2012.

${ }^{59}$ COMISIÓN EUROPEA: https://ec.europa.eu/echo/where/africa/share-horn-of-africa_en [Consultado el 24 de septiembre, 2019].

60 THE FOOD CRISIS PREVENTIVE NETWORK: http://www.food-security.net/en/topic/global-alliance-for-resilience-agir/ [Consultado el 25 de septiembre, 2019]

${ }^{61}$ JOSEPH, Jonathan, "The EU in the Horn of Africa: Building Resilience as a Distant Form of Governance" en Journal of common market studies, $\mathrm{n}^{\circ}$ 52.2, 20I4, pp. 285-30I.

${ }^{62}$ JUNCOS, Ana E, "Resilience as the new EU foreign policy paradigm: a pragmatist turn?" en European security, n 26. I, 20I7, pp. I-I8.

${ }^{63}$ SHIFERAW, B., et al. "Managing vulnerability to drought and enhancing livelihood resilience in sub-Saharan Africa:Technological, institutional and policy options" en Weather and Climate Extremes, n 3, 2014, pp. 67-79.

${ }^{64}$ THE VIA CAMPESINA NOTEBOOKS: https://viacampesina.org/en/wp-content/uploads/sites/2/2010/09/Multiple-crisis-for-small-scalefarmers.compressed.pdf [Consultado el 30 de Septiembre, 2019]. 
En los países más afectados como Somalia, Etiopia y Kenia no hay organizaciones miembros en Via Campesina. Los resultados de AGIR-Sahel son modestos: su página-web recoge solo tres ejemplos específicos de Senegal y cuatro generales en Gambia.

En suma, una política de resiliencia a distancia, pragmática o simple no parece ser suficiente dada la complejidad del problema de la sequía y hambruna en el Sahel, no solo en su aspecto natural, agrícola o alimenticio, sino también en lo que se refiere al sistema institucional, de poder y de relaciones internacionales. El problema necesita una mayor proactividad exterior y una mayor participación de los colectivos de agricultores. Por el contrario, el movimiento social transnacional de agricultura ecológica y soberanía alimentaria es un ejemplo de promoción global de resiliencia positiva, apoyada indirectamente en iniciativas públicas, como son algunas políticas medioambientales de la UE. ¿Se puede sugerir que las ONGD podrían promover la resiliencia positiva de una manera más efectiva?

\section{Hacia una visión societal y civil de la seguridad, el desarrollo y la resiliencia}

Sobre la discusión teórica y los ejemplos expuestos anteriormente se presenta una propuesta teórica de la seguridad y la resiliencia en el contexto de los países periféricos, de renta baja o en desarrollo, que son los países con mayor nivel de conflictos violentos y los más afectados por desastres naturales. Se proponen dos conceptos: intervención civil y empoderamiento autógeno.

\section{I. Seguridad, intervención civil y ONGH}

¿Qué tipo de intervención internacional es más adecuada y efectiva para promover la seguridad y la paz en los conflictos? ¿Qué instituciones y organizaciones deberían ejecutar esa intervención? Chambler ${ }^{65}$ contrasta el tradicional alto intervencionismo occidental con el bajo intervencionismo de los discursos de seguridad y resiliencia. El declive del intervencionismo estatal-militar de los países centrales es concebido como una práctica neoliberal que no responde a la obligación moral de "responsabilidad de proteger" a las personas vulnerables de la sociedad civil. Para Chambler, el enfoque de la seguridad humana como proyecto de creación de resiliencia y empoderamiento de los grupos vulnerables es un marco en el que pueden encajar las dos formas de intervencionismo a modo de justificación complementaria, y toda intervención militar de protección debería ir orientada a potenciar la resiliencia de la población vulnerable. Este es un discurso legítimo y correcto, pero en la realidad empírica resulta difícil observar los efectos prácticos. La intervención pacificadora y aseguradora exterior puede ser de muchas maneras, orientaciones y prioridades. $A$ continuación, se presenta la noción de intervención civil.

En el caso de guerras, conflictos, e incluso de desastres naturales de alta escala,la intervención pública suele estar liderada por los gobiernos y agencias estatales, con sus diversos recursos financieros, militares, información, etc.- en colaboración con grandes empresas especializadas en logística, transporte, etc. Sin embargo, en la realidad la intervención estatal y empresarial suele tener efectos indirectos no deseados, principalmente por los intereses escondidos que en muchas ocasiones acompañan a los gobiernos, los estados y las grandes empresas. Por el contrario, la intervención de los actores civiles, principalmente la $\mathrm{ONU}$ y $\mathrm{ONGH}$, es un tipo de intervención más imparcial y neutral, con menos efectos negativos o no intencionados. Este tipo de intervención se puede denominar intervención civil o público-civil.

${ }^{65}$ CHANDLER, David, “Resilience and human security:The post-interventionist paradigm” en Security dialogue, n 43.3, 2012, pp. 2 I 3-229. 
La intervención civil, liderada por las ONG y organizaciones civiles, coordinadas y supervisadas por la ONU y agencias intergubernamentales, tiene más probabilidades de efectivad y legitimidad en el largo plazo. Dada la difícil cooperación interestatal en las relaciones internacionales actuales, la ONU, desde una mayor autonomía de gestión, debe seguir potenciando la incorporación y la participación de las ONG locales y globales en sus decisiones y programas, evolucionando gradualmente hacia una unión global, o de orientación global, de naciones-estado y sociedades civiles democráticas ${ }^{66}$. Las $\mathrm{ONGH}$ deben ver reconocido su importante papel en la promoción de la seguridad de la población ${ }^{67}$. En suma, la idea de intervención civil se refiere especialmente a la labor que las ONGH pueden realizar para la creación y el desarrollo de la seguridad societal, entendida como ausencia de violencia o riesgo. La seguridad humana integral debe basarse en una estable seguridad civil y poblacional, sustentada localmente.

\subsection{Empoderamiento autógeno y ONGD}

Si la resiliencia proactiva y positiva puede ser estimulada exteriormente, puede considerarse como un fenómeno de empoderamiento autógeno. Autógeno significa generación propia, frente a endógeno que es generación desde el interior. El empoderamiento autógeno es un empoderamiento diferente al exógeno y al endógeno. Se asume que el empoderamiento exógeno suele estar abocado al fracaso dada la falta de proximidad cultural y de legitimidad sociopolítica. El empoderamiento endógeno suele ser difícil de iniciarse en comunidades o colectivos con un relativo bajo nivel de dinamismo y con estructuras de poder excesivamente jerárquicas y verticales. En esencia, el empoderamiento autógeno se refiere al impulso o la promoción externa para crear las precondiciones o factores previos que facilitan empoderamiento endógeno.

¿Quiénes pueden ser los actores o factores externos que promueven esas precondiciones? La construcción de las precondiciones o factores propulsores del empoderamiento endógeno pueden ser facilitada por las ONGD, a través de dos mecanismos principales.Primero,generando una mayor y mejor interacción social organizada y semiorganizada entre los diferentes miembros de la comunidad o sociedad, a través de diferentes tipos de encuentros abiertos, amplios y participativos, priorizando la participación de las mujeres para buscar el necesario equilibrio de género. La teoría sociológica interaccionista argumenta que la interacción social organizada entre personas es el mecanismo y la experiencia básica para la construcción de la conciencia, la identidad y las creencias colectivas, como precondiciones para los intereses comunes y la prosperidad colectiva. Segundo, a través de la formación de personas o grupos promotores del empoderamiento endógeno, que una vez capacitadas promuevan las precondiciones desencadenantes de la participación y la autoconciencia colectiva, y el desarrollo inclusivo.

El empoderamiento autógeno puede también ser considerado como una fuente de resiliencia societal positiva, en la medida en que promueve la conciencia colectiva y la interacción social y grupal, en donde se transmiten los aprendizajes relativos a la superación de adversidades. Las ONGD pueden por tanto jugar ese papel de constructores del empoderamiento autógeno. Las capacidades de resiliencia positiva de un colectivo humano deben fundamentarse en un empoderamiento asumido como propio y auténtico. En resumen, el empoderamiento civil y

\footnotetext{
${ }^{66}$ MARTENS, Kerstin, “Civil society and accountability of the United Nations” en SCHOLTE, Jan Aaart (ed.), Building global democracy? Civil society and accountable global governance, Cambridge University Press, $201 \mathrm{I}$.

67 SAUQUILLO, Francisca, La otra cara... op cit.
} 
societal sentido como propio y auténtico es una precondición para la prosperidad colectiva.

\section{Conclusiones}

Tras revisar críticamente la literatura sobre los conceptos de seguridad y resiliencia se han relacionado con la intervención internacional en regiones o países periféricos por parte de países centrales, y con las políticas de ayuda al desarrollo, con atención a la actuación de la UE.

Respecto a la seguridad, se ha relacionado con las políticas de intervención en situaciones de conflicto y riesgo. Desde el análisis de los casos de los conflictos de Congo y Colombia, se ha argumentado que, para ayudar a solucionar situaciones de conflicto violento a gran escala, la intervención internacional más efectiva en el largo plazo tiene lugar cuando la ayuda se dirige a la sociedad civil y local, en donde se aprehende la complejidad del conflicto y se construyen las bases de una reconciliación y paz. Se ha enfatizado la importancia de la seguridad civil o societal, en el que la paz o ausencia de violencia deviene de una alta participación local. Sobre esta base analítica, se ha propuesto el concepto de intervención civil, el cual enfatiza que en casos de conflictos o desastres naturales la intervención liderada por ONGH y coordinada por organismos gubernamentales dentro del marco de la ONU suele ser real o potencialmente más efectiva, especialmente en el medio y largo plazo. La seguridad o ausencia de violencia se fundamenta en la construcción local de una narrativa comprensiva y en acuerdos de pacificación y seguridad, en las que las ONG pacifistas y humanitarias locales tienen un papel central. Las ONGH de los países centrales pueden:a) promover las ONG locales para impulsar la paz y la seguridad, y b) coordinar la participación de las poblaciones locales en la resolución de los conflictos.

Respecto a la resiliencia, se ha diferenciado entre resiliencia tradicional o positiva. Se ha relacionado con el desarrollo y las políticas de ayuda al desarrollo, ilustrado con el papel de la UE respecto a la compleja crisis del Sahel en África, y el movimiento global de agricultura ecológica. Se ha subrayado que la resiliencia positiva puede ser promovida exteriormente de forma directa o indirecta, $y$ en este sentido se ha enfatizado que el empoderamiento local puede ser estimulado por las ONGD. Sobre esta base analítica, se ha propuesto el concepto de empoderamiento autógeno, que se refiere al proceso de empoderamiento local ayudado por elementos externos pero percibido como proceso propio. Es una forma de empoderamiento con un alto nivel intersubjetivo de autonomía, o de resiliencia proactiva y positiva. Las ONGD pueden promover las precondiciones para este tipo de empoderamiento, a través de dos mecanismos: a) mejorar la interacción social organizada y semiorganizada en la comunidad o sociedad local, y b) producir promotores de la participación y el desarrollo inclusivo y participativo, formando personas locales con aspiración de transformación de su comunidad.

Dos consideraciones finales sobre el alcance global y político de los argumentos. Primero, enfatizar el enfoque global de las cuestiones de seguridad y resiliencia. Desde la perspectiva del Antropoceno o Capitaloceno sobre el orden humano natural planetario hay que tener en cuenta que cada sociedad organizada en estados-naciones tiene efectos directos e indirectos sobre el orden humano-natural tanto el propio como el global común. Sin intención de ser alarmista, si se quiere quizá evitar el surgimiento de tensiones o incluso conflictos violentos por cuestiones naturales relacionados con el cambio climático, es necesario aumentar la conciencia colectiva global. Segundo, la dimensión política y moral. Los conceptos de seguridad-intervención civil y 
de empoderamiento autógeno, además de poder ser elementos de una teoría del desarrollo de la seguridad humana y la prosperidad colectiva de una sociedad dentro de sus relaciones internacionales, apelan a la responsabilidad de los países centrales para ayudar a los países periféricos, muchos de ellos excolonias de imperios occidentales. En la época de los crecientes retos globales, los países centrales del mundo deben promover la construcción y el desarrollo de sociedades civiles democráticas en los países periféricos, y una forma de conseguir ese objetivo puede ser a través de la intervención civil y del empoderamiento autógeno.

\section{Bibliografía}

ABIEW, Francis K.,"NGO-military relations in peace operations" en International Peacekeeping, n I0.I, 2003, pp. 24-39.

ABRAHAMSEN, Rita yWILLIMAS, Michael C., Security beyond the state:Private security in international politics, Cambridge University Press, 2010.

Acción Humanitaria; concepto y evolución, en Diccionario de Acción Humanitaria y Cooperación al Desarrollo de HEGOA: http://www.dicc.hegoa.ehu.es/listar/mostrar/I [Consultado el 25 de septiembre de 2019].

AHEDO, Manuel, "Una sociología histórica y global de la construcción del estado racionalizado de la modernidad internacional" en Relaciones Internacionales, $n^{\circ}$ 38, 2018, pp. II-29.

ALTIERI, Miguel Angel y TOLEDO, Victor Manuel, “The agroecological revolution in Latin America: rescuing nature, ensuring food sovereignty and empowering peasants" en The Journal of Peasant Studies, $n^{\circ}$ 38.3, 20I I, pp. $587-612$.

ARNOULD, Valerie y VLASSEROOT, Koen, "EU policies in the DR Congo: Misaligned ambitions", en KALDOR, Mary, RANGELOV, lavor y Sabine SELCHOW (eds.), EU Global Strategy and Human Security, Routledge, Londres, 2018, pp. 85-102.

AUTESERRE, Sverine, Peaceland: Conflict resolution and the everyday politics of international intervention, Cambridge University Press, 2014

AUTESSERRE, Séverine, "Dangerous tales: Dominant narratives on the Congo and their unintended consequences" en African Affairs, $\mathrm{n}^{\circ}$ II I .443, 201 2, pp. 202-222.

AUTESSERRE, Séverine, "International peacebuilding and local success:Assumptions and effectiveness" en International Studies Review, n 19.1, 2017, pp. II4-132.

BARNES, Barry, The elements of social theory, UCL Press, Londres, 1995.

BECK, Ulrich, World at risk, Polity, Londres, 2009.

BORDÁ, Sandra y GÓMEZ, Santiago,"La Internacionalización del proceso de paz de la Habana: del aislacionismo a la contención" en Tickner A. y Bitar S. (eds.), Nuevos enfoques para el estudio de las relaciones internacionales en Colombia, Universidad de los Andes, Bogotá D.C., 2017, pp. 223-238.

BRASSET, James y VAUGHAN-WILLIAMS, Nick, "Security and the performative politics of resilience: Critical infrastructure protection and humanitarian emergency preparedness" en Security Dialogue, $n^{\circ}$ 46. I, 20 I5, pp. $32-50$.

BURGUESS, J. Peter, The Future of Security Research for the Social Science and Humanities, European Science Foundation, Estrasburgo, 2014.

BURGUESS, J. Peter, "Security Studies" en GUILLAUME, Xavier y BILGIN, Pinar (eds.), Routledge handbook of international political sociology, Routledge, Londres, 2016, pp. 233-244.

BUZAN, Barry, People, states \& fear. An agenda for international security studies in the post-cold war era, The Open University, Londres, 1982/1991.

BUZAN, Barry, WÆVER Ole y der WILDE, Jaap, Security: a new framework for analysis, Boulder, Lynne Rienner Publishsers, 1998.

CASTAÑEDA, Dorly, ¿Qué significan los laboratorios de paz para la unión europea?» en Colombia internacional, $n^{\circ}$ 69, 2009, pp. I62-179.

CASTAÑEDA, Dorly, The European Union in Colombia: Learning how to be a peace actor, L'Institut de Recherche Stratégique de l'École Militaire, Paris, 2012.

COMISIÓN EUROPEA: https://ec.europa.eu/echo/where/africa/share-horn-of-africa_en [Consultado el 24 de septiembre, 2019].

CONTRERAS, Lara, “La resiliencia de la teoría a la práctica: un enfoque para romper el círculo del hambre”, Cap. 7 en el Informe del Observatorio de la Acción Humanitaria, IECAH, Madrid, 2012.

CHANDLER, David, "Resilience and human security:The post-interventionist paradigm” en Security dialogue, $n^{\circ} 43.3$, 2012, pp. 213-229.

CHANDLER, David,"International state-building and the ideology of resilience” en Politics, $n^{\circ}$ 33.4, 2013, pp. 276-286.

CHANDLER, David y COAFFEE, Jon, The Routledge handbook of international resilience, Routledge, Londres, 2016.

CUJABANTE, Ximena, "La comunidad internacional y su participación en los procesos de paz en Colombia" en Equidad 
y Desarrollo, $n^{\circ} 26,2016$, pp. 207-222.

DAVISON, John y AMMANN, Klaus, "New GMO regulations for old: determining a new future for EU crop biotechnology" en GM crops \& food, n 8.I, 2017, pp. 13-34.

DE CONING, Cedric, "From peacebuilding to sustaining peace: Implications of complexity for resilience and sustainability" en Resilience, $n^{\circ}$ 4.3, 2016, pp. I66-18I.

DEL ARENAL, Celestino y SANAHUJA, José Antonio (coord.), Teorías de las Relaciones Internacionales, Tecnos, Madrid, 2015

DONAIS, Timothy, "Empowerment or Imposition? Dilemmas of Local Ownership in Post $\square$ Conflict Peacebuilding Processes" en Peace \& Change, ${ }^{\circ}$ 34.I, 2009, 3-26.

DONAIS, Timothy, Peacebuilding and local ownership: Post-conflict consensus-building, Routledge, Londres, 2012.

FALKNER, Robert,"'The political economy of 'normative power' Europe: EU environmental leadership in international biotechnology regulation" en Journal of European Public Policy, $\mathrm{n}^{\circ}$ I4.4, 2007, pp. 507-526.

FISAS, Vicente, Introducción a los procesos de Paz, Escola de Cultura de Pau, Barcelona, 2010.

GAHAMA, Joseph, "Une cinquantaine d'années de conflits et de violences dans la région des Grands Lacs africains" en LUMUMBA-KASONGO Tukumbi \& GAHAMA Joseph (eds.), Peace, Security and Post-conflict Reconstruction in the Great Lakes Region of Africa, COIDERSA, Dakar (Senegal), 2017, pp. 9-28.

GONZALEZ, Catherine, "Iniciativas de paz en Colombia” en Civilizar: Ciencias Sociales y Humanas, $\mathrm{n}^{\circ}$ 10.18, 2010, pp. 35-53.

GUBBELS, Peter, "Escaping the Hunger Cycle: Pathways to resilience in the Sahel" en Oxfam Policy and Practice: Agriculture, Food and Land, $\mathrm{n}^{\circ}$ II.6, 201 I, pp. 165-288.

GRAN, Guy, Development by People; citizen construction of a just world, Praeger, Santa Barbara (EUA), 1983.

HERNÁNDEZ DELGADO, Esperanza,“"Negociaciones de paz en Colombia:una mirada en perspectiva de construcción de paz" en Papel Político, $n^{\circ} 21.1,2016$, pp. 35-56.

JOSEPH, Jonathan, "Resilience as embedded neoliberalism: a governmentality approach" en Resilience: International Policies, Practices and Discourses, $n^{\circ}$ I.I, 20I3, pp. 38- 52.

JOSEPH, Jonathan, "The EU in the Horn of Africa: Building Resilience as a Distant Form of Governance" en Journal of common market studies, $\mathrm{n}^{\circ}$ 52.2, 20I4, pp. 285-30I.

JUNCOS, Ana E.,"Resilience as the new EU foreign policy paradigm: a pragmatist turn?" en European security, $\mathrm{n}^{\circ} \mathrm{26.I}$, 2017, pp. I-18.

KALDOR, Mary, RANGELOV, lavor y SELCHOW, Sabine (eds.), EU Global Strategy and Human Security, Routledge, Londres, 2018.

KORTEN, David C., Getting to the 2 Ist century:Voluntary action and the global agenda, Kumarian Press, West Hartford (EUA), 1990.

KRÜGER, Marco, "Building Instead of Imposing Resilience: Revisiting the Relationship Between Resilience and the State" en International Political Sociology, n I3.I, 20 I8, pp. 53-67.

LANGA MARTINEZ, Laura,"El camino a la resiliencia. La llave para la convergencia de enfoques y actores" Documento I5/20I3, IECAH, Madrid, 2013.

LE BILLON, Philippe, Wars of Plunder: Conflicts, Profits and the Politics of Resources, Oxford University Press, Oxford, 2014.

MAHECHA, Jimena, "Iniciativas locales de paz: tres casos desde la resistencia civil para la reflexión" en Ciencia Política, $n^{\circ}$ 13.26, 2018, pp. 153-18I.

MARTENS, Kerstin, "Civil Society and accountability of the United Nations" en SCHOLTE, Jan Aaart (ed.), Building global democracy? Civil society and accountable global governance, Cambridge University Press, 20I I, pp. 42-57.

MARTIN, Mary y OWEN Taylor, "The Second Generation of Human Security: Lessons from the UN and EU Experience" en International Affairs, $\mathrm{n}^{\circ} 86.1,2010$, pp. 2 II -224.

MARTINUSSEN, John Degnbol, Society, state \& market. A guide to competing theories of development, MSO, Danish Association for International Cooperation, Copenhague, 1997 (re-publicado en 2015 por HSRC Publishers).

MEAGUER, Kate, "The strength of weak states? Non $\square$ state security forces and hybrid governance in Africa" en Development and Change, $\mathrm{n}^{\circ} 43.5,2012$, pp. 1073-I I0I.

MUÑOZ, Francisco A. y Jorge BOLAÑOS CARMONA (eds.), Los habitus de la paz. Teorías y prácticas de la paz imperfecta, Editorial Universidad de Granada, Granada, 20II.

MURRAY,Tonita, "Police-building in Afghanistan:A case study of civil security reform" en International Peacekeeping, $\mathrm{n}^{\circ}$ 14.I, 2007, pp. 108-126.

NEWMAN, Edward, "Critical Human Security Studies" en Review of International Studies, n 36, 20I0, pp. 77-94.

NÚNEZ, Jesús, HAGERAATS, Balder y REY, Francisco, "Seguridad Humana, recuperando un concepto necesario" en Cuadernos del IECAH, n. 7, Madrid, 2007.

PÉREZ DE ARMIÑO, Karlos, La vinculación emergencia-desarrollo en el marco del nuevo humanitarismo. Reflexiones y propuestas, Coordinadora de ONG para el Desarrollo, Madrid, 2002.

PÉREZ DE ARMIÑO, Karlos y MENDIA AZKUE, Irantzu (eds.), Seguridad Humana. Aportes críticos al debate teórico y político, Editorial Tecnos, Madrid, 2013.

REID, Julian, "Interrogating the Neoliberal Biopolitics of the Sustainable Development-Resilience Nexus" en International Political Sociology, $\mathrm{n}^{\circ}$ 7.4, 2013, pp. 353-367.

ROBERTSON, Alexander F., People and the State:An Anthropology of Planned Development, Cambridge University Press, 
1984.

RUTHERFORD, Ken, BREM, Stefan, Matthew, Richard Anthony y Matthew, Richard (eds.), Reframing the agenda: the impact of NGO and middle power cooperation in international security policy, Greenwood Publishing Group, Connecticut (EUA), 2003.

SANAHUJA, José Antonio y SCHÜNEMANN, Julia, "El nexo seguridad-desarrollo: entre la construcción de la paz y la securitización de la ayuda", en PÉREZ DE ARMIÑO, Karlos y MENDIA AZKUE, Irantzu (eds.), Seguridad Humana. Aportes críticos al debate teórico y político, Editorial Tecnos, Madrid, 20I3, pp. I7-70.

SAUQUILLO, Francisca, "La otra cara de la resolución de conflictos. Las ONG" en Cuadernos de estrategia, $n^{\circ}$ I47, 2010, pp. $141-173$.

SEN, Amartya, Development as freedom, Anchor Books, Nueva York (EUA), 1999.

SHIFERAW, Bekele,TESFAYE, Kindie, KASSIE, Menale,ABATE,Tsedeke, PRASANNA, B. M.y MENKIR Adebe,"Managing vulnerability to drought and enhancing livelihood resilience in sub-Saharan Africa:Technological, institutional and policy options" en Weather and Climate Extremes, $n^{\circ}$ 3, 20I4, pp. 67-79.

THE FOOD CRISIS PREVENTIVE NETWORK: http://www.food-security.net/en/topic/global-alliance-for-resilienceagir/ [Consultado el 25 de septiembre, 2019].

THE VIA CAMPENSINA NOTEBOOKS: https://viacampesina.org/en/wpcontent/uploads/sites/2/2010/09/Multiplecrisis-for-small-scale-farmers.compressed.pdf [Consultado el 30 de Septiembre, 2019].

TUKALA, Célestin Tshimande,"Etats des Grands Lacs et privatisation de la sécurité: quel enjeu pour la gouvernance?," en LUMUMBA-KASONGO Tukumbi \& GAHAMA Joseph (eds.), Peace, Security and Post-conflict Reconstruction in the Great Lakes Region of Africa, COIDERSA, Dakar (Senegal), 2017, pp. 299-326.

UNIÓN EUROPEA: https://eeas.europa.eu/topics/eu-global-strategy_en [Consultado el 25 de septiembre de 2019].

UNIÓN EUROPEA, “Responsible trade in minerals from high-risk or conflict areas": https://eur-lex.europa.eu/legalcontent/EN/LSU/?uri=CELEX:320 I7R082 I \&qid=|542740800246 [Consultado el 27 de septiembre de 2019]

VAUGHAM-WILLIAMS, Nick, Critical security studies:An introduction, Routledge, Londres, 2010.

VERDES-MONTENEGRO ESCANÉZ, Francisco J.,"Securitización: agendas de investigación abiertas para el estudio de la seguridad" en Relaciones internacionales, $\mathrm{n}^{\circ}$ 29, 2015, pp. I I I-I32.

WALSH-DILLEY, Marygold,WOLFORD, Wendy y MCCARTHY, James, "Rights for resilience: food sovereignty, power, and resilience in development practice" en Ecology and Society, n' ${ }^{\circ}$ I. I, 2016: www.jstor.org/stable/26270348 [Consultado el 25 de septiembre de 2019].

W/AVER, Ole, BUZAN, Barry, KELSTRUP, Morten y LEMAITRE, Pierre, Identity, migration and the new security agenda in Europe, Pinter Publishers, Reino Unido, 1993.

YOUNGS, R. La fusión entre seguridad y desarrollo: ¿Otro estancamiento europeo?, FRIDE, Madrid, 2007. 


\section{RELACIONES INTERNACIONALES}

Revista académica cuatrimestral de publicación electrónica Grupo de Estudios de Relaciones Internacionales (GERI)

Universidad Autónoma de Madrid, España

https://revistas.uam.es/relacionesinternacionales

ISSN 1699 - 3950

f facebook.com/RelacionesInternacionales

3. twitter.com/RRInternacional

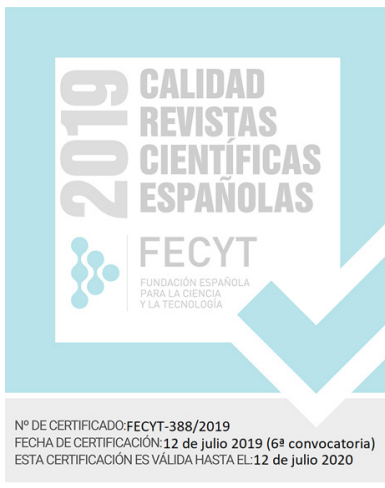

